\title{
Prevention and reversal of chronic diseases: A Protocol
}

\author{
Ronald N. Kostoff ${ }^{1}$
}

\author{
AFFILIATION \\ 1 Independent Consultant, Gainesville, United States
}

CORRESPONDENCE TO

Ronald N. Kostoff. Independent Consultant, 13500 Tallyrand Way, Gainesville, VA 20155, United States. E-mail: rkostoff@gmail.com ORCID ID: https://orcid.org/0000-0002-4434-2147

KEYWORDS

chronic kidney disease, contributing factors, peripheral arterial disease, Alzheimer's disease, chronic disease prevention, chronic disease reversal

Received: 4 November 2021, Revised: 3 December 2021, Accepted: 4 December 2021

Public Health Toxicol 2021;1(2):10

https://doi.org/10.18332/pht/144538

\section{ABSTRACT}

For a decade, our research group has been developing protocols to prevent and reverse chronic diseases. The present article outlines the lessons we have learned from both conducting the studies and identifying common patterns in the results. The main product of our studies is a five-step treatment protocol to reverse any chronic disease, based on the following systemic medical principle: at the present time, removal of cause is a necessary, but not necessarily sufficient, condition for restorative treatment to be effective. Implementation of the five-step treatment protocol to reverse any chronic disease is as follows: 1 ) Obtain a detailed medical and habit/exposure history from the patient; 2) Administer qualitative and quantitative performance and behavioral tests to assess the severity of symptoms and performance measures; 3) Administer laboratory tests (blood, urine, imaging, etc.); 4) Identify and eliminate contributing factors ( $\mathrm{CFs}$ ) to the chronic disease of interest; and 5) Implement treatments for the chronic disease of interest. This individually-tailored chronic disease treatment protocol can be implemented with the data available in the biomedical literature now. It is general and applicable to any chronic disease that has an associated substantial research literature (with the possible exceptions of individuals with strong genetic predispositions to the disease in question or who have suffered irreversible damage from the disease). To prevent any chronic disease, eliminate those factors that have been identified to contribute to the onset of the target disease.

Our recent studies on common CFs between selected chronic diseases and COVID-19 show that many of the CFs that enable or exacerbate the selected chronic diseases studied also enable or exacerbate COVID-19. While further studies are required, it appears that the toxicology-based methods we have developed for preventing or reversing chronic diseases may also be applicable to preventing infectious diseases, such as COVID-19.

\section{INTRODUCTION}

Concept for preventing and reversing chronic diseases 'Noncommunicable diseases (NCDs) kill 41 million people each year, equivalent to $71 \%$ of all deaths globally ${ }^{1}$. While the per capita mortality rate for infectious diseases has declined precipitously over the past century, the per capita mortality rate for non-communicable diseases has remained roughly constant ${ }^{2}$.

Whether this constant per capita mortality trend for non-communicable diseases will continue into the future is highly questionable. The three chronic diseases we studied (Chronic Kidney Disease, CKD; Alzheimer's Disease, AD; and Peripheral Neuropathy/Peripheral Arterial Disease, PN/
PAD) that served as a basis for our prevention and reversal protocol for any chronic disease have a strong toxicology component. There have been many potentially harmful and effectively un-regulated high-technology additions to the environment in the past few decades (e.g. non-ionizing radiation, from cell phones and $\mathrm{Wi}-\mathrm{Fi}$; inadequately tested vaccines; agricultural chemicals, etc.). Because of latency delays, it is difficult to link the disease and potentially harmful environmental factors that may have occurred decades earlier. As, for example, our AD study ${ }^{3}$ showed, the adverse impact of recent potentially harmful environmental and dietary additions on AD biomarkers and symptoms ominously portends increased incidence and prevalence of 
$\mathrm{AD}$ in the future. This holds true for all three chronic diseases we studied.

Many of the toxic stimuli that are CFs to AD also contribute to a myriad of other serious diseases ${ }^{4}$. Many of these diseases can be fatal, and may not have the multi-decadal latencies associated with AD. Thus, these lethal diseases serve to cull out people who would have been high-risk candidates for $\mathrm{AD}$ had they lived. This culling out of high-risk individuals artificially depresses and masks the real incidence of AD, had these high-risk people survived. This line of reasoning is applicable to the other chronic diseases we studied.

It was important to understand better what was driving the disparity in mortality trends between communicable and non-communicable diseases, and to ascertain whether the text mining approaches we developed for discovering new treatments for disease could help reverse the trend. We found that these three chronic diseases examined were being addressed in the clinic and the literature by mainly treating aberrations in the host systems related to their symptoms. For example, AD was addressed by treating amyloid beta, tau phosphorylation, and cholinergic neuron death, and PAD was addressed by treating plaque buildup and blood flow problems. The toxicology components underlying the CFs that produced these aberrations/symptoms were not being addressed.

We concluded that treatments were insufficient as long as the factors that contributed to the disease were still operable. The unrecognized toxicology component had to be addressed and eliminated. As a result, we developed a systemic medical principle that, in its latest incarnation, states: at the present time, removal of cause is a necessary, but not necessarily sufficient, condition for restorative treatment to be effective. The principle has two caveats: irreversible damage has not been done, and there is not an overwhelming genetic predisposition to the disease in question. However, in most cases, we will not know whether irreversible damage has been done or whether there is an effective genetic predisposition to the disease until essentially all the important CFs have been eliminated. This principle is general, and applicable to prevention and reversal of any disease. The methodology that has been developed based on this principle is general, and applicable to any disease as well. To prevent any disease, the foundational causes shown by the literature to underlie the disease symptoms and biomarkers need to be identified and removed as comprehensively, thoroughly, and rapidly as possible. To reverse any disease (given the above caveats), the preventive steps above need to be implemented, and treatments to reverse the disease progression need to be applied.

The efficacy of the methodology for preventing and reversing any disease depends on how thoroughly the foundational causes, treatments, biomarkers, and symptoms of the disease of interest have been identified. As will be shown in this article, a wide spectrum of existing foundational CFs has been identified for the chronic diseases we have studied, and a wide spectrum of existing treatments and symptoms and biomarkers has been identified as well. Combining these results allows development of a treatment protocol that can be tailored to individual patients and applied to any chronic disease. Most importantly, this treatment protocol (based on the systemic medical principle described above) is available with the information at our disposal today.

Further, our recent studies on common CFs between COVID-19 and selected chronic diseases ${ }^{5,6}$ show that many of the CFs that enable or exacerbate COVID-19 also enable or exacerbate the selected chronic diseases studied (Inflammatory Bowel Disease, IBD; Gastrointestinal Cancer, GIC). Thus, while additional studies are required for a more complete picture, it appears that the methods we have developed for preventing or reversing chronic diseases may also be applicable to preventing infectious diseases.

The information presented in the present article is a condensation and update of a 2019 monograph $^{7}$. There is much detail presented in the monograph, especially the appendices, and the reader will be directed toward the appropriate sections of the monograph if this level of detail is desired.

\section{METHODOLOGICAL APPROACH}

\section{Concept feasibility}

Currently, the protocol we have developed is a concept. What evidence exists to demonstrate its feasibility? An example is shown for $\mathrm{AD}$, but it can be extrapolated to the other chronic diseases as well. In 2017, two books were published on reversing early-stage $\mathrm{AD}^{8,9}$. All the authors were credible neurologists, with long-standing experience in treating neurodegenerative diseases.

One of the authors, Bredesen ${ }^{10}$, is an AD researcher/ clinician who has shown that 'reversal of cognitive decline in patients with early Alzheimer's disease or its precursors, MCI (mild cognitive impairment) and SCI (subjective cognitive impairment)' is obtainable today ${ }^{10}$. Basing his approach on optimizing metabolic parameters, Bredesen used a combination of eliminating some potential AD CFs, substituting positive health practices, and adding dietary supplements to achieve his $\mathrm{AD} / \mathrm{MCI} / \mathrm{SCI}$ reversal results.

Sherzai and Sherzai ${ }^{9}$ are AD researchers/clinicians who claim to have had very positive results with patients based on: 'lifestyle intervention as the cure for cognitive decline'. Their lifestyle modification approach (based on addressing their assumed four main pathways to AD: inflammation, oxidation, glucose dysregulation, lipid dysregulation) has five main components: nutrition, exercise, stress management, adequate sleep, and mental challenges.

The approaches of Bredesen ${ }^{10}$ and Sherzai \& Sherzai ${ }^{9}$ can be viewed as one 'footprint' of the more general systemic medical principle for preventing or reversing disease described in the current article. Our approach is not constrained by hypotheses based primarily on symptoms/ 
pathological mechanisms. We use symptoms, pathological mechanisms, and other abnormal AD characteristics as a guidepost to identify causes to be eliminated and treatments to be implemented for individual patients. Our approach is based on cause and effect, as evidenced in the premier biomedical literature. We identify as many AD biomarkers and symptoms as exist in the literature, and then relate adverse changes in the values of these AD biomarkers and symptoms to potential underlying foundational causes. Preferably, the research findings will identify the biological mechanisms that link a foundational cause to its impact(s) on AD biomarkers and symptoms. However, even in the absence of identifying such mechanisms, the linkage is retained. We also relate beneficial changes in the values of these AD biomarkers and symptoms to potential treatments, again, whether or not the biological mechanisms that link treatments to positive impacts have been identified.

Since our approach incorporates the positive aspects of the approaches of Bredesen ${ }^{10}$ and Sherzai \& Sherzai ${ }^{9}$, but is far more extensive, we would expect (with some degree of confidence) the results from our approach to be at least as beneficial as theirs, and possibly be effective for the higher stages of $\mathrm{AD}$ as well.

\section{Concept cost impacts}

There are two major benefits that would result from widescale implementation of our concept: improved health and longevity, and greatly reduced healthcare costs. The latter are addressed in this section.

There are many schemes being proposed in the US today to reduce the massive level of healthcare costs. They go by the names of 'Medicare for All', 'single-payer', 'public option', 'universal healthcare', etc. Essentially all these proposals are 'book-keeping' schemes. They do little to reduce the people, infrastructure, and procedures involved in healthcare. Rather, they focus on who is going to pay the bills, and which scheme will have the greatest political impact.

None of these schemes will do very much to reduce total healthcare costs. We will still need a myriad of: specialists, support personnel, diagnostics, drugs, therapies, nursing homes, etc., under the mainstream medical approach used today. Paperwork may be reduced, and some savings may result from eliminating the insurance companies, but the bulk of healthcare costs will remain. In fact, bringing in more people to healthcare system coverage under these proposals will result in increased total costs, unless drastic changes in healthcare procedures are adopted.

The only way to reduce healthcare costs substantially is to keep people out of the medical system in the first place, wherever possible. Procedures need to be implemented that will minimize peoples' interactions with the medical system. Rather than manipulate accounting schemes to reduce healthcare costs a few percent, eliminate (as broadly as possible) those exposures and substances that are known to contribute to disease.
As the remainder of this article will show, we already know most of the factors that contribute to noncommunicable diseases. For the chronic diseases we have studied over the past decade, there are hundreds of CFs for each disease, most of which are not addressed under existing medical care. Additionally, in some recent toxicology studies we have performed to support the protocols, we have found there are orders of magnitude differences between exposure limits set by Federal regulators and exposures shown to cause damage in the biomedical literature ${ }^{11}$.

Significant reduction of healthcare costs requires two major steps. First, the medical profession needs to place far more emphasis on reducing CFs to disease in their treatment protocols, along the lines suggested in this article. This step also requires educating patients on the importance of this approach for their healing, and would greatly reduce peoples' interactions with the medical system. Second, government at all levels needs to bring the regulated exposure limits in line with the findings in the medical literature. This includes limits on chemical exposures, radiation exposures (especially non-ionizing radiation), biotoxin exposures, etc., taking into account potentially synergistic effects due to combinations of a myriad of toxic stimuli exposures ${ }^{12,13}$. Implementation of the above two steps would lead to far more health improvement and cost reductions than any of the accounting schemes under present consideration.

\section{PROTOCOL IMPLEMENTATION}

Our previous work on using text mining to treat diseases had focused almost exclusively on discovering new treatments for diseases using Literature-Related Discovery (LRD - an off-shoot of Literature-Based Discovery) developed about a decade ago ${ }^{14-19}$. We then began to expand our text mining approach to identifying CFs as well as treatments. We would identify not only potential new CFs and treatments for the disease of interest, but would also include identifying known $\mathrm{CFs}$ and treatments for the disease of interest.

The initial chronic disease we examined was $\mathrm{CKD}^{20}$. We developed more powerful LRD techniques to identify potential new treatments, and were able to apply these techniques to identify potential new CFs. Equally important, as it turned out, we developed new text mining approaches to identify comprehensively the known treatments and CFs for CKD. Appendix 1 of Kostoff $^{7}$ outlines the text mining approaches used for the CKD study; the detailed text mining approaches for each of the chronic diseases are presented in later appendices.

The results from the CKD study were very surprising because of their magnitude and breadth. About $800+\mathrm{CFs}$, about $800+$ treatments, and almost 400 biomarkers/ symptoms were identified from the existing CKD literature. We believe many more of each were possible, especially those appearing infrequently in the CKD literature. In addition, almost 100 new CFs and almost 100 new treatments were identified as discovery from the non-CKD literature. Again, 
much more discovery was possible, since only very simple queries were used, and the analysis of what was retrieved was arbitrarily terminated due to resource limitations.

Our next published study did not focus on a specific disease. After the CKD study, it became clear that a much more comprehensive understanding of the broader impacts of potentially toxic substances on all diseases simultaneously was required before moving ahead to develop protocols to prevent and reverse other specific diseases. An approach was conceived that would identify 'all' CFs to 'all' diseases. This methodology is presented in Appendix 2 of Kostoff'. The comprehensive study was a massive effort that was limited severely by available resources. The final product was an eBook entitled 'Pervasive Causes of Disease'4. It identified about 8000 causes spread over about 4000 diseases. About 800 of the causes were deemed 'pervasive', meaning they impacted at least an arbitrarily-selected threshold number of diseases. The relationships tended to be many-to-one and one-to-many. One cause could impact many diseases, and many causes could impact one disease.

The cause-disease matrix was very sparse; only a relatively few of the 32 million cells in the matrix had values. Far more cause-disease linkages should be expected. The various biological systems are interconnected (neural, immune, endocrine, circulatory, etc.), and an adverse impact of a substance on one of these systems would be expected to have a ripple effect on the other intersecting systems. A more comprehensive and adequately-resourced study could have uncovered many more cause-disease relationships from the existing literature. However, even if all the cause-disease relationships had been identified from the existing biomedical literature, the matrix still may have been relatively sparse. In order for a cell to have an entry, the underlying research would have had to be funded, conducted, and published. In Chapter 9 of the Pervasive Causes of Disease eBook ${ }^{4}$, reasons are discussed why some of this research never sees the light of day, especially for topics that have commercial/political/military sensitivity. Also, in the design and conduct of the research, the researchers do not include the thousands of biomarkers that would cover all these diseases in any one experiment or study. They usually measure a handful of such biomarkers in any one study, selected mainly because of mechanism assumptions, and their findings are typically limited to one or a few diseases.

Our next published study focused on $\mathrm{AD}^{3}$. Based on lessons learned from the CKD study and the Pervasive Causes of Disease study, we developed a more streamlined approach, and were more selective in the number of different text mining approaches used to identify CFs, treatments, and symptoms/biomarkers. This streamlined methodology is shown in Appendix 3 of Kostoff'. We also developed a more formal treatment protocol for preventing and reversing $\mathrm{AD}$. As in the CKD study, we found many hundreds of CFs, treatments, and biomarkers/symptoms, and could have identified many more of each by using additional approaches.
In the AD biomedical literature, there are at least $20+$ hypotheses (e.g. amyloid hypothesis, tau hypothesis, etc.). We assumed all had some validity, and selected three or four biomarkers and symptoms to represent each hypothesis in the diagnostic steps of the protocol. All the AD hypotheses identified were centered around AD pathology, specifically, general biomarker abnormalities and symptoms (e.g. amyloid plaques, tau hyperphosphorylation, oxidative stress, inflammation, etc.) associated with AD. The typically high-technology treatments that accompany these hypotheses focus on removing/suppressing these pathological symptoms, rather than removing the causes of these symptoms. These treatments (in the absence of comprehensive cause removal) are not efficacious because they violate the systemic medical principle that forms the basis of our methodology.

Interestingly, there are no foundational causes-based hypotheses of AD, e.g. the Deficient-Diet Hypothesis, the Iatrogenic Hypothesis, the Sedentary Lifestyle Hypothesis, the Radiation Exposure Hypothesis, the Toxic Chemical Exposure Hypothesis, etc. There are many articles in the literature: 1) questioning the validity of each of the abovelisted pathology-based AD hypotheses, and 2) showing the deficiencies in their associated treatments for reversing AD.

The strategy of identifying symptoms as pathological mechanisms that must be suppressed or removed for healing is a mainstay of Western Medicine. However, another perspective is to view these symptoms as having two basic functions: serve as a warning signal that dysfunction exists and actions need to be taken to remove the cause of this dysfunction, and serve as a protective mechanism.

There are many examples in the biomedical literature supporting the concept of disease symptoms as warning signals and protective mechanisms, as shown in Table 1.

Additionally, in his 2017 book, Bredesen ${ }^{8}$ states: 'Alzheimer's disease is actually a protective response to, specifically, three different processes: inflammation, suboptimal levels of nutrients and other synapse-supporting molecules, and toxic exposures'. Other AD researchers have drawn similar conclusions. If Bredesen's view (that the AD symptoms serve as a protective response against more serious damage) is correct, then the mainline drug-based $\mathrm{AD}$ treatment approach of suppressing these symptoms without removing the foundational causes that underlie these symptoms comprehensively in parallel: 1) effectively removes the protective shield offered by these symptoms, and thereby 2) exacerbates the progression of AD.

Our most recent study of protocols for chronic disease prevention and reversal focused on $\mathrm{PN} / \mathrm{PAD}^{26}$. We improved our techniques for identifying existing $\mathrm{CFs}$, treatments, and biomarkers/symptoms. As a result, we identified $800+\mathrm{CFs}$, $1000+$ treatments, and 1000+ biomarkers and symptoms. The PN/PAD methodology is contained in Appendix 4 of Kostoff'. Again, identifying far more items was possible with a well-funded study. 


\section{Table 1. Examples of disease symptoms as protective mechanisms}

1. 'The down-regulation of energy metabolism in AD is a protective response of the neurons to the reduced level of nutrient and oxygen supply in the microenvironment ${ }^{21}$.

2. 'Neurofibrillary tangle formation as a protective response to oxidative stress in Alzheimer's Disease ${ }^{22}$.

3. 'Autophagy is a protective response to the oxidative damage to endplate chondrocytes in intervertebral disc ${ }^{23}$.

4. 'Loss of appetite in the acute phase of illness is indeed an adaptive, protective response that improves cell recycling (autophagy) and detoxification'24.

5. 'Cataract is a self-defence reaction to protect the retina from oxidative damage'25.

\section{A note about the very large number of existing treatments identified in all three chronic disease prevention and reversal studies}

In all three, the treatments identified covered research over the past two to three decades. Treatments that have 'failed' in human clinical trials are not excluded. The reason for retaining these 'failed' treatments is as follows. On reading thousands of abstracts on laboratory experiments and clinical trials of potential chronic disease treatments, it became apparent that:

1) in vitro experiments typically performed on cells tend to have reasonably positive outcomes, at least for those articles that surface in the peer-reviewed published literature;

2) in vivo experiments typically performed on rodents (but other small animals as well) tend to also have reasonably positive outcomes, albeit somewhat less than in vitro experiments; and

3) When these potential treatments reach the human clinical trial stage, especially the later phases, the success rates plummet.

The explanation for this discrepancy given most often is the species difference. Humans are different from rodents etc., and their physiological responses to stimuli are different as well. However, the toxic experiential and exposure background differences between humans who live in the real-world sea of myriad toxic exposures and experimental animals who live in the very controlled environment of the laboratory are rarely, if ever, discussed.

For the three chronic diseases studied, there were many hundreds of potential CFs identified (ranging from Lifestyle to Occupational/Environmental exposures). For a given individual, some causes have happened in the past, and are no longer happening, but their damage trail remains. Other causes are ongoing, have caused damage, and continue to cause damage.
Why would anyone expect a human being with such a toxic history to respond to a potential treatment the same way that a laboratory animal raised in a controlled environment would respond to that treatment? Furthermore, why would anyone expect a human being with such a toxic history to respond to a potential treatment the same way that another human being without such a toxic burden would respond to that treatment?

Consider the example of T. Wahls, an MD, who was able to reverse her own case of Multiple Sclerosis (MS) ${ }^{27}$. She used two main types of treatments: lifestyle changes (mainly dietary) to reverse the MS and neuromuscular electrical stimulation (NMES) to reverse the damage resulting from MS. It was only when her diet achieved substantial improvement that the NMES produced positive effects.

While T. Wahls' experience represents only one data point, it is a very powerful data point. Consider its implications. Suppose a clinical trial were conducted to evaluate the potential for NMES to reverse the damage from MS. Suppose further that Wahls' dietary-dominant CF to MS and her reaction to NMES were typical of the participants in such a clinical trial. If the participants did not address their diet (with the rigor shown by Wahls) during the clinical trial, they would not respond positively to the NMES (as was the case for Wahls initially). The trial would be interpreted as a failure of NMES. However, in this hypothetical example, the NMES ineffectiveness is not the reason for the clinical trial's lack of success. Failure to remove the cause of the disease and subsequent damage is the problem. Failure to remove cause (of the three chronic diseases) as a reason for the very limited success of myriad treatments in the clinical trials of the past three decades cannot be ruled out.

That is why even so-called 'failed' treatments were included in the studies of the three chronic diseases. It cannot be stated conclusively which treatments failed because: 1) they were intrinsically ineffective, or 2) their beneficial effects were overwhelmed by the strong negative effects of the ongoing causes remaining operable. In fact, it is unknown whether comprehensive, timely, and thorough removal of the relevant CFs for each of the three diseases by themselves would have obviated the need for many of these treatments.

There was no single text mining/information retrieval method that extracted the full spectrum of CFs or treatments or biomarkers from the biomedical literature for any of the diseases studied. Each of the methods used provided new information. There were four categories of approaches used to identify CFs, treatments, and biomarkers, and future studies should select at least one approach from each category for comprehensiveness. The details of these methodologies are presented in section 2A of Kostoff?

\section{CFs to disease}

Many hundreds of foundational CFs were identified for each disease examined. Appendix 5 of Kostoff ${ }^{7}$ contains an 
extensive presentation of the CFs to AD. It is similar to the structure and quantity of CFs to the other chronic diseases. Appendix 6 also contains a taxonomy of impacts from AD foundational CFs. This structure is similar to the impacts from other chronic disease CFs.

Some of the foundational CFs that are pervasive not only to the specific chronic diseases examined, but to many others as well, include:

- Dietary - high fat $\operatorname{diet}^{28,29}$, high salt $\operatorname{diet}^{30,31}$, refined carbohydrates $^{32,33}$, advanced glycation end products ${ }^{34,35}$, high cholesterol $\operatorname{diet}^{36,37}$, and vitamin deficiency ${ }^{38-45}$;

- Substance abuse - smoking ${ }^{46,47}$, and alcohol ${ }^{48,49}$;

- Iatrogenic - antibiotics ${ }^{50-52}$, anesthetics ${ }^{53,54}$, antipsychotics $^{55,56}$, radiotherapy ${ }^{57,58}$; and

- Occupational/environmental - solvents ${ }^{59-61}$, endocrine disruptors ${ }^{62,63}$, pollutants/pollution ${ }^{64-66}$, pesticides ${ }^{67-70}$, heavy metals ${ }^{71-80}$, and combined exposure ${ }^{81-84}$.

Many of these pervasive CFs are under individual control (such as diet, substance abuse, drugs to some extent), while others (such as occupational/environmental exposures) may not be obvious without extensive and complex testing, and require government regulation for control.

\section{Treatments for disease}

Many hundreds of treatments were identified for each disease examined. Appendix 7 of Kostoff ${ }^{7}$ contains a comprehensive list of the treatments for $\mathrm{AD}$, and Appendix 8 contains a table of treatment benefits for AD. These are similar to the quantity of treatments and treatment impacts for the other chronic diseases examined.

\section{Biomarkers and symptoms}

Many hundreds of biomarkers, and somewhat less symptoms, were identified for each disease examined. Appendix 9 of Kostoff ${ }^{7}$ contains a table of biomarkers for PN/PAD, followed by a table of symptoms for PN/PAD. These are similar to the types and quantity of biomarkers and symptoms for the other chronic diseases examined.

\section{Patterms in CFs to disease}

The dominant finding from the three chronic disease studies and the large Pervasive Causes of Disease study was the large number and wide spectrum of CFs to each disease. The challenge was to identify any underlying structures in this voluminous data, and ascertain whether any messages could be gleaned that would support development of protocols to prevent and reverse these diseases.

It became clear that a first-order categorization of the CFs could be achieved mechanistically. The CFs were divided into six categories (with some overlap): Lifestyle, Iatrogenic, Biotoxins, Occupational/Environmental, Psychosocial/Socioeconomic, and Genetics. The last category was not pursued further, since the thrust of the CFs was foundational/modifiable CFs (those tangible causes over which we had, in theory, some control). The categories proved useful for presenting results.

The next question was whether there were a few latent categories that would explain the larger structure. It soon became evident that the bulk of CFs to disease were: 1) the products of modern and semi-modern technology, especially technology that was effectively unregulated, and from 2) the spinoffs of this modern technology (such as sedentary living, staring at computer screens all day, etc.). The problem can be stated summarily as follows.

\section{Direct technology}

Direct technology (the degree of direct impact of technology on foundational causes) plays a strong role in Lifestyle, Iatrogenic, and Occupational/ Environmental foundational causes. In addition, through its impact on the immune and other critical systems, modern technology may play a role in whether exposure to bacteria and viruses translates to symptoms and diseases. Modern technology impacts the growing, processing, and preparation of foods, and many of the adverse effects identified in the Pervasive Causes of Disease eBook can be traced back to the use (or misuse) of technology in the food cycle. The Iatrogenic adverse effects of modern technology result mainly from the hightechnology-based drugs, surgery, diagnostics, and therapy that characterize much of modern medicine today. The Occupational/Environmental adverse effects result mainly from the employment of modern technology in commerce, the environment, and the workplace.

\section{Inadequate regulation}

Inadequate Regulation is coupled strongly to the introduction of high technology in all aspects of life. Many of the problems with foods derive from relatively unregulated chemicals, materials, and other contaminants entering the food supply during agriculture and animal husbandry. Many of the Occupational/Environmental exposures arise from relatively unregulated harmful substances entering the workplace and the environment. This is especially true in less developed countries, but occurs in more developed countries as well. Many of the Iatrogenic problems could be traced to drugs, diagnostics, therapies, and other procedures entering practice with insufficient front-end long-term testing (especially testing on humans), and inadequate evaluation of side-effects.

Two major aspects of Inadequate Regulation revolve around insufficient safety: inadequate safety data gathering, and inadequate safety testing. Much of the adverse impact data gathering tends to be from passive surveillance systems, where response rates can be an order of magnitude (or more) less than real-world incidence rates. Pre-market testing, in many cases, suffers from inadequate sample sizes, unrepresentative samples, insufficient long-term testing, and insufficient combination testing to identify potential synergistic effects. Insufficient long-term testing on humans is particularly troubling, since many serious diseases such 
as AD may have decadal latency periods from specific toxic stimuli. Transgenerational effects could not be excluded without appropriate long-term testing.

Additionally, results from animal testing (which could be long-term from the perspective of many short-lived animals used in testing) do not necessarily translate to human outcomes. First, there is a species difference, and impacts on one species do not necessarily carry over to the same types of impacts on another species. Second, laboratory animals are raised in relatively pristine environments, and subjected to a very few toxic substances during studies on disease CFs. Conversely, humans experience many of the CFs identified in Pervasive Causes of Disease, and the synergy from these combinations would not have been replicated in the laboratory animal testing.

\section{Setting priorities for preventing and reversing chronic} diseases

Our most recent chronic disease study was for PN/PAD. Its core literature was not nearly as large as that of the CKD and AD studies, yet substantially more CFs, treatments, and biomarkers were found relative to the CKD and AD studies. We attribute that to the experience gained in identifying these quantities from having performed the CKD and $\mathrm{AD}$ studies, and to the improvements made in the identification algorithms.

It is clear that future studies of major chronic diseases will generate at least the levels of numbers obtained in the PN/ PAD studies (on the order of 1000 each of CFs, treatments, and biomarkers), and perhaps far more if the studies are resourced adequately. Given these voluminous numbers, some type of priority will need to be assigned to: 1) CFs to eliminate, 2) biomarkers to be used for diagnostics, 3) treatments to be implemented, and 4) make the treatment protocol feasible for clinicians.

\section{CFs Priorities}

CFs differ in myriad ways, but two are of interest here: ease of identification, and ease of elimination. Many aspects of diet, recreational substance use, amount of exercise, etc., are examples of CFs that are easy to identify. Exposure to environmental and workplace toxic substances require targeted measurements, and are far more difficult to identify. Dietary substances, recreational drugs, etc., are (in theory) easy to eliminate. Toxic exposures not being measured are impossible to eliminate. Other toxic exposures in the residential area and in the workplace that one cannot avoid (because they cannot afford to move or change jobs) are difficult to eliminate.

Our three chronic disease studies resulted in development of a five-step protocol for preventing and reversing chronic diseases. The protocol is shown in summary form for $\mathrm{AD}$ in Table 2, but applies to any chronic disease.

For chronic disease, one or more abnormalities in different test results will emerge from steps 1-3 (Table
2). In theory, the CFs can be matrixed against biomarkers, symptoms, etc., to ascertain which factors are contributing heavily to the abnormalities. Unfortunately, the results are not that clear-cut. Consider a matrix of CFs against biomarkers, specific and general. For general biomarkers, such as inflammation and oxidative stress, there could be hundreds of CFs that impact these general biomarkers. For some specific biomarkers, such as excess mercury, the CF linkage is much clearer and easier to eliminate. While there would probably be health benefits if the hundreds of CFs to inflammation and oxidative stress were eliminated, changes on that order of magnitude are probably not realistic. Additionally, if a person has to be instrumented to ascertain whether he/she is being exposed to the unmeasured CFs, that would require a long and expensive process in today's environment.

In the $\mathrm{AD}$ and $\mathrm{PN} / \mathrm{PAD}$ studies, an alternative prioritization approach was recommended. The concept is to initiate the $\mathrm{CF}$ elimination process by eliminating those factors: 1) identified in the study, 2) easiest to eliminate, and 3) under one's control (more or less). We called this eliminating the 'low-hanging fruit'. These CFs had been identified in all three chronic disease studies, and in the Pervasive Causes of Disease study. They will probably appear in most major chronic disease studies, but that could be easily checked when these studies are completed. Table 3 contains the latest incarnation of the 'low-hanging fruit' CFs.

Even in the 'low-hanging fruit' list, the first half are much more straight-forward to identify and eliminate than the second half.

\section{Table 2. Five-step treatment protocol to reverse AD}

1. Obtain a detailed medical and habit/exposure history from the patient.

2. Administer written and clinical performance and behavioral tests to assess the severity of the higher-level symptoms and degradation of executive functions.

3. Administer laboratory tests (blood, urine, imaging, etc.).

4. Eliminate ongoing AD CFs.

5. Implement AD treatments.

\section{Biomarkers priorities}

There is a gross mismatch between the thousand or so biomarkers identified in our latest chronic disease studies (and expected in future chronic disease studies) and the number of biomarkers typically used in clinical practice for testing. The identified biomarkers need to be reduced by almost two orders of magnitude (with today's analytical instrumentation and costs) to be acceptable in clinical practice. Experience from the $\mathrm{AD}$ and PN/PAD studies has shown that the relatively modest numbers of general 
biomarkers identified could serve as a starting point for the reduction process. In the AD study, the hypotheses selected to start the culling process were, for the most part, congruent with the general biomarkers identified (e.g. inflammation, oxidative stress, apoptosis, neurodegeneration, etc.). In the PN/PAD study, the main general biomarkers identified were used to start the culling process.

Once the general biomarkers were selected, they were then matrixed against the specific biomarkers, and about three specific biomarkers were selected to represent each general biomarker. This would result in somewhere between 50 and 100 specific biomarkers to be used in the diagnostics tests. If that proves to be too many for some clinicians, then

\section{Table 3. 'Low-hanging fruit' recommendations}

1. Curb the dietary excesses, and remove the dietary deficiencies, identified in the CFs list, the medical questionnaire, and the lab tests.

2. Eliminate food additives to the extent knowable and possible, including those dietary excesses that derive from food additives (excessive fat, sugar, salt).

3. Minimize high temperature cooking and the subsequent increases in advanced glycation end products from certain susceptible foods, heterocyclic amines, acrylamide, and polycyclic aromatic hydrocarbons.

4. Reverse the sedentary behavior patterns identified.

5. Remove the foundational impediments to better sleep.

6. Eliminate the use of 'recreational' drugs, including smoking and excessive alcohol.

7. Eliminate the use of medicinal drugs shown in the potential CFs list, unless these drugs are absolutely necessary.

8. Minimize exposures to some hydrocarbons, such as n-hexane, methyl-n-butyl ketone, carbon disulfide, acrylamide, ethylene oxide, trichloroethylene, kerosene, polycyclic aromatic hydrocarbons (including those found in smoke), etc.

9. Minimize exposures to some neurotoxic solvents, especially organic solvents.

10. Minimize inhalation and ingestion exposures to pesticides, herbicides, insecticides, and fungicides.

11. Minimize exposures to heavy metals in food, in water, and in the air.

12. Minimize exposure to particulates, especially air pollution

13. Minimize exposures to ionizing radiation and nonionizing non-visible radiation (such as cell phones, cell towers, Wi-Fi, smart meters, etc.).

14. Minimize chronic stress (mental/emotional/ psychological). two specific biomarkers could be selected rather than three to represent each general biomarker, and the numbers would drop by about a third.

\section{Treatment priorities}

In the chronic disease studies performed so far, there tended to be relatively few treatments universally used by the mainstream medical community. Realistically, we would expect that trend to continue. However, in all cases we examined, there tended to be significant numbers of articles addressing adverse effects ('side-effects') of each of these treatments. They are not without risk. Additionally, when these types of treatments (shown to have some risk when tested in isolation) are combined with other similar treatments, enhanced risk could result from synergies or additive effects.

The approximately 1000 treatments identified in the latest chronic disease study covered a very wide spectrum, and varied significantly in their levels of risk. There was a core of low-risk treatments that impacted most, if not all, of the general biomarkers selected. Elimination of a CF could be viewed as a low-risk treatment. Substitution of a health-promoting habit for a health-degrading habit could be viewed as a treatment. There are many other types of low-risk treatments possible, and they do not involve drugs, radiation, or surgery. The last could be utilized if the previous prove to be insufficient. Similar to the recommended prioritization of CF selection starting with the 'low-hanging fruit', our recommended prioritization of treatments starts with low-risk treatments (identified in the study). Table 4 presents some of these low-risk treatments.

\section{Caveats on diet}

1. Many toxic/harmful substances enter the food supply during all phases of food growth, distribution, and processing. While foods should be selected to maximize the amounts of healing nutrients identified above, care must be taken to minimize the level of toxic additions to the food in parallel.

2. Low-temperature cooking should be used to minimize production of AGEs and other harmful products (nitrosamines, polycyclic aromatic hydrocarbons, and acrylamides) during the cooking process.

3. Only low-mercury wild-caught fish should be used; these tend to be smaller fish, lower on the food chain.

4. Grass-fed animals with no exogenous growth hormones or antibiotics should be used, if possible, since these harmful products could be passed through to the consumer.

5. For fruits and vegetables normally eaten with skin, those that have not been sprayed with harmful pesticides and other toxic chemicals should be used.

6. Heavy metals are a CF for many diseases, especially neurodegenerative diseases. One source of heavy metal bioaccumulation in the body is through the food supply. 


\section{Table 4. Lowest-risk treatments}

1. Exercise (such as aerobic exercise, walking, resistance training, treadmill, calisthenics, stretching, balancing).

2. Sleep improvement (such as quiet environment, minimal light, minimal food before bedtime, maintain regular sleep schedule).

3. Stress reduction (such as tai chi, yoga, massage, aromatherapy, acupuncture, acupressure, sensory stimulation, physiotherapy, massage, reflexology, meditation).

4. Diet - choose foods high in:

- Polyphenols (such as cloves, star anise, capers, curry powder, ginger, cinnamon, peppermint, oregano, sage, rosemary, thyme, basil, cocoa, tea, red wine, chokeberries, elderberries, blueberries, plums, cherries, black currants, blackberries, strawberries, raspberries, grapes, flaxseeds, celery seeds, chestnuts, hazelnuts, pecans, almonds, walnuts, olives, artichokes, chicory, red onion, spinach, broccoli, apples, pomegranates, peaches, apricots, olive oil, canola oil), especially flavonoids (such as apples, blueberries, strawberries, red grapes, cabbage, broccoli, onions, capers, dark chocolate, cocoa, tea, red wine), isoflavones/genistein (such as soybeans, natto, tempeh, tofu, miso), and anthocyanins (such as blackberries, black currants, blueberries, strawberries, cranberries, eggplant, cherries, prunes, raisins, and the darker versions of raspberries, cabbage, plums, radish, grapes, plums, apples, beans, beets, cabbage, onions, pears, wines)

- Unrefined carbohydrates (such as whole grains, legumes, fruits, and uncooked vegetables)

- DHA/omega-3 fatty acid (such as salmon, herring, mackerel, anchovy, sardine, trout, shark, swordfish, mussel, sea bass, pollock, whiting, flounder, sole, lobster, halibut, carp, oyster, crab, mullet, tuna, perch, snapper, shrimp, octopus)

- Vitamin B12/Folate (such as meat [beef liver, lamb, beef], fish [sardines, mackerel, salmon], dairy [feta cheese, cottage cheese], eggs, legumes [chickpeas, fermented soy, pinto beans, lentils], fruit [banana, avocado], vegetables [spinach, parsley, broccoli, beets, turnip, asparagus])

- Vitamin C (such as fruits [guavas, acerola cherry, kiwifruit, rose hips, strawberries, oranges, papayas], vegetables [bell peppers, broccoli, tomatoes, snow peas, kale])

- Vitamin D (such as fish [sardines, salmon, mackerel, tuna], liver [beef, calf, cod liver oil], dairy [milk, yogurt]; most importantly, sunlight on exposed skin)

- Vitamin E (such as seeds [sunflower seeds, pumpkin seeds], nuts [almonds, hazelnuts, pine nuts], fish [abalone, salmon, trout], fruit [avocado, mango, kiwifruit], vegetables [red peppers, turnip greens, spinach, chard, squash, broccoli])

- Lycopene (such as tomatoes, guavas, watermelon, papaya, grapefruit)

- Oleic acid (such as nuts [almonds, peanuts, pecans, cashews, pistachios, hazelnuts] seeds [sesame, sunflower], avocados, olives, and vegetable oils [safflower, almond, olive, sesame, sunflower])

- Luteolin (such as dried oregano, celery seed, hot peppers, peppermint, sage, rosemary, juniper berries, thyme, radicchio, Chinese celery)

- Quercetin (such as capers, lovage leaves, elderberry juice, dock leaves, radish leaves, arugula, dill weed, coriander, and fennel, cilantro, banana peppers, juniper berries, oregano, onions, carob flour, radicchio, red leaf lettuce, onions, watercress, raw, asparagus, kale, okra, cocoa powder, chia seeds)

- Sulforaphane (such as broccoli sprouts, broccoli, cauliflower, kale, brussels sprouts, cabbage, collards, arugula, turnips)

- Resveratrol (such as red wine, red grapes, peanut butter, pistachios, cocoa powder, dark chocolate, strawberries, blueberries, bilberries, cranberries)

- Epigallocatechin-3-gallate (such as green tea, black tea, carob powder, apples, blackberries)

Heavy metals can occur naturally in the soil in which food is grown, they can concentrate abnormally in soils from nearby industrial pollution or from precipitation of air pollution, they can preferentially absorb in different types of food, and, depending on the type of food, can be absorbed from the food processing and manufacturing process. Any of the above foods selected for chronic disease prevention or treatment/reversal purposes should have heavy metal concentrations as low as possible.

\section{DISCUSSION}

The central thesis of our treatment protocol for chronic diseases is that CF elimination is a prime requirement for 
the possibility of preventing and reversing chronic disease. However, the inverse is also true. CF enhancement is a prime requirement for developing and exacerbating chronic disease.

Unfortunately, there are myriad ways in which CFs to disease are imposed on the population by government mandates, loose regulations, and other means. Effectively, government is promoting and, in some sense, mandating the expansion of chronic disease among its population. Section 2C of Kostoff ${ }^{7}$ shows a detailed example of how the government is effectively mandating exposure to harmful levels of non-ionizing radiation, and how OSHA is effectively mandating exposure to harmful levels of toxic stimuli through lax regulations of these chemicals and materials.

\section{CONCLUSIONS}

We have developed a general protocol for preventing and reversing any chronic disease. It is based on the following systemic medical principle: 'at the present time, removal of cause is a necessary, but not necessarily sufficient, condition for restorative treatment to be effective.' The principle has two caveats: irreversible damage has not been done, and there is not an overwhelming genetic predisposition to the disease in question.

The protocol starts with a medical history and myriad biomarker and performance tests to identify aberrant symptoms and biomarker levels. It then identifies factors that contribute to these aberrant symptoms and biomarker levels. For disease prevention, adoption of these factors should be minimized. For disease reversal, these factors should be eliminated as deeply, widely, and rapidly as possible.

The protocol was tailored for three different chronic diseases: CKD, AD, and PAD/PN. In each case, hundreds of known and repurposed CFs were identified as candidates for elimination, and many known and repurposed treatments were identified as well. The repurposed treatments and CFs were identified using our LRDI process.

There is much overlap of CFs among the three diseases, and less overlap among the treatments. Our recent publications on CFs common to two other chronic diseases (IBD, GIC) and COVID-19 imply the existence of a unity between chronic and infectious diseases. This unified theory of infectious-chronic diseases further implies that switching from a symptom-based coordinate system to a CF-based coordinate system for preventing and treating/reversing diseases can produce synergistic benefits for eliminating both types of disease in any individual.

\section{REFERENCES}

1. Noncommunicable diseases: Key facts. World Health Organization; 2021. April 13, 2021. Accessed December 3, 2021. https://www.who.int/en/news-room/fact-sheets/ detail/noncommunicable-diseases

2. Hansen V, Oren E, Dennis LK, Brown HE. Infectious Disease
Mortality Trends in the United States, 1980-2014. JAMA. 2016;316(20):2149-2151. doi:10.1001/jama.2016.12423

3. Kostoff RN, Porter AL, Buchtel HA. Prevention and reversal of Alzheimer's disease: treatment protocol. Georgia Institute of Technology; 2018. Accessed December 3, 2021. https:// smartech.gatech.edu/handle/1853/59311

4. Kostoff RN. Pervasive Causes of Disease. Georgia Institute of Technology; 2015. Accessed December 3, 2021. http://hdl. handle.net/1853/53714

5. Kostoff RN, Briggs MB, Kanduc D, Shores DR, Kovatsi L, Vardavas AI, Porter AL. Common contributing factors to COVID-19 and inflammatory bowel disease. Toxicol Rep. 2021;8:1616-1637. doi:10.1016/j.toxrep.2021.08.007

6. Kostoff RN, Briggs MB, Kanduc D, et al. Contributing factors common to COVID-19 and gastrointestinal cancer. Oncol Rep. 2022;47(1):16. doi:10.3892/or.2021.8227

7. Kostoff RN. Prevention and Reversal of Chronic Disease: Lessons learned. Georgia Institute of Technology; 2019. Accessed December 3, 2021. http://hdl.handle. net/1853/62019

8. Bredesen DE. The End of Alzheimer's: The first program to prevent and reverse cognitive decline. Avery; 2017.

9. Sherzai D, Sherzai A. The Alzheimer's solution: a breakthrough program to prevent and reverse the symptoms of cognitive decline at every age. HarperOne; 2017.

10. Bredesen DE. Reversal of cognitive decline: a novel therapeutic program. Aging. 2014;6(9):707-717. doi:10.18632/aging.100690

11. Kostoff RN. OSHA Permissible Exposure Limits (PELs) are too Permissive. Georgia Institute of Technology; 2018. Accessed December 3, 2021. http://hdl.handle.net/1853/60067

12. Kostoff RN, Goumenou M, Tsatsakis A. The role of toxic stimuli combinations in determining safe exposure limits. Toxicol Rep. 2018;5:1169-1172. doi:10.1016/j.toxrep.2018.10.010

13. Kostoff RN, Aschner M, Goumenou M, Tsatsakis A. Setting safer exposure limits for toxic substance combinations. Food Chem Toxicol. 2020;140:111346. doi:10.1016/j.fct.2020.111346

14. Kostoff RN. Literature-related discovery (LRD): Potential treatments for cataracts. Technol Forecast Soc Change. 2008;75(2):215-225. doi:10.1016/j.techfore.2007.11.006

15. Kostoff RN. Literature-related discovery: Potential treatments and preventatives for SARS. Technol Forecast Soc Change. 2011;78(7):1164-1173. doi:10.1016/j.techfore.2011.03.022

16. Kostoff RN. Literature-Related Discovery (LRD): Potential treatments for Parkinson's Disease. Technol Forecast Soc Change. 2008;75(2):226-238. doi:10.1016/j.techfore.2007.11.007

17. Kostoff RN, Los LI. Literature-related discovery techniques applied to ocular disease: a vitreous restoration example. Curr Opin Ophthalmol. 2013;24(6):606-610. doi:10.1097/ICU.0b013e3283654def

18. Kostoff RN, Block JA, Stump JA, Johnson D. Literaturerelated discovery (LRD): Potential treatments for Raynaud's Phenomenon. Technol Forecast Soc Change. 2008;75(2):203214. doi:10.1016/j.techfore.2007.11.005

19. Kostoff RN, Briggs MB, Lyons T. Literature-related discovery 
(LRD): Potential treatments for Multiple Sclerosis. Technol Forecast Soc Change. 2008;75(2):239-255. doi:10.1016/j.techfore.2007.11.002

20. Kostoff RN, Patel U. Literature-related discovery and innovation: Chronic kidney disease. Technol Forecast Soc Change. Feb 2015;91:341-351. doi:10.1016/j.techfore.2014.09.013

21.Sun J, Feng X, Liang D, Duan Y, Lei H. Down-regulation of energy metabolism in Alzheimer's disease is a protective response of neurons to the microenvironment. J Alzheimers Dis. 2012;28(2):389-402. doi:10.3233/JAD-2011-111313

22. Nunomura A, Takeda A, Moreira PI, et al. Neurofibrillary Tangle Formation as a Protective Response to Oxidative Stress in Alzheimer's Disease. In: Maccioni RB, Perry G, eds. Current Hypotheses and Research Milestones in Alzheimer's Disease. Springer; 2009:103-113. doi:10.1007/978-0-387-87995-6_9

23. Chen K, Lv X, Li W, et al. Autophagy Is a Protective Response to the Oxidative Damage to Endplate Chondrocytes in Intervertebral Disc: Implications for the Treatment of Degenerative Lumbar Disc. Oxid Med Cell Longev. 2017;(4041768):1-9. doi:10.1155/2017/4041768

24. Schütz P, Bally M, Stanga Z, Keller U. Loss of appetite in acutely ill medical inpatients: physiological response or therapeutic target? Swiss Med Wkly. 2014;144:w13957. doi:10.4414/smw.2014.13957

25. Wegner A, Khoramnia R. Cataract is a self-defence reaction to protect the retina from oxidative damage. Med Hypotheses. 2011;76(5):741-744. doi:10.1016/j.mehy.2011.02.013

26. Kostoff RN. Prevention and Reversal of Peripheral Neuropathy/Peripheral Arterial Disease. Georgia Institute of Technology; 2019. Accessed December 3, 2021. http://hdl. handle.net/1853/61865

27. Wahls T. TEDxIowaCity: Minding your mitochondria. Youtube. November 30, 2011. Accessed December 3, 2021. https:// www.youtube.com/watch?v=KLjgBLwH3Wc

28. Elahi M, Motoi Y, Shimonaka S, et al. High-fat diet-induced activation of SGK1 promotes Alzheimer's disease-associated tau pathology. Hum Mol Genet. 2021;30(18):1693-1710. doi:10.1093/hmg/ddab115

29. Madkhali HA, Ganaie MA, Ansari MN, et al. Red Yeast Rice Mitigates High-Fat Diet Induced-Obesity Related Vascular Dysfunction in Wistar Albino Rats. Biointerface Res Appl Chem. 2021;11(6):14290-14303. doi:10.33263/briac116.1429014303.

30.Fyfe I. High-salt diet promotes Alzheimer diseaselike changes. Nat Rev Neurol. 2020;16(1):2-3. doi:10.1038/s41582-019-0289-7

31. Babaer D, Amara S, Ivy M, et al. High salt induces P-glycoprotein mediated treatment resistance in breast cancer cells through store operated calcium influx. Oncotarget. 2018;9(38):25193-25205. doi:10.18632/oncotarget.25391

32. Butler MJ, Deems NP, Muscat S, Butt CM, Belury MA, Barrientos RM. Dietary DHA prevents cognitive impairment and inflammatory gene expression in aged male rats fed a diet enriched with refined carbohydrates. Brain Behav
Immun. 2021;98:198-209. doi:10.1016/j.bbi.2021.08.214

33.Zanol JF, Niño OMS, da Costa CS, Freitas-Lima LC, Miranda-Alves L, Graceli JB. Tributyltin and high-refined carbohydrate diet lead to metabolic and reproductive abnormalities, exacerbating premature ovary failure features in the female rats. Reprod Toxicol. 2021;103:108-123. doi:10.1016/j.reprotox.2021.06.004

34. Roza N, Quadros K, Esteves A, et al. P-321: Advanced Glycation End-Products Are Related With Cortical Quality And Increased Risk For Bone Fractures In Chronic Kidney Disease Patients. J Bone Miner Res. 2020;35(S1):148-148. Accessed December 3, 2021. https://asbmr.onlinelibrary. wiley.com/doi/epdf/10.1002/jbmr.4206

35. Omofuma 00, Peterson LL, Turner DP, et al. Dietary Advanced Glycation End-Products and Mortality after Breast Cancer in the Women's Health Initiative. Cancer Epidemiol Biomarkers Prev. 2021;30(12):2217-2226. doi:10.1158/1055-9965.EPI-21-0610

36. Kapelouzou A, Katsimpoulas M, Kontogiannis C, et al. A HighCholesterol Diet Increases Toll-like Receptors and Other Harmful Factors in the Rabbit Myocardium: The Beneficial Effect of Statins. Curr Issues Mol Biol. 2021;43(2):818-830. doi:10.3390/cimb43020059

37. Seenak P, Kumphune S, Malakul W, Chotima R, Nernpermpisooth N. Pineapple consumption reduced cardiac oxidative stress and inflammation in high cholesterol diet-fed rats. Nutr Metab (Lond). 2021;18(1):36. doi:10.1186/s12986-021-00566-z

38. Chandra S, Thomas S, Nangia A, Singh R, Pathania OP. Association Between Endemic Vitamin D Deficiency in India and High Prevalence of Poor-Prognosis Triple-Negative Breast Cancer: a Cross-Sectional Study. Indian J Surg. 2021. doi:10.1007/s12262-021-03065-w

39. Rofei M, Morelli C, Riondino S, et al. 492P Vitamin D deficiency in metastatic colorectal cancer (mCRC) worsens survival and correlates with significant peripheral inflammatory/immune cell changes. Ann Oncol. 2021;32:S574. doi:10.1016/j.annonc.2021.08.1011

40. Vitamin D deficiency causes cardiac dysfunction mediated by TRPC6_complete data. US Environmental Protection Agency. doi:10.23719/1503424

41. Mauro AG, Kraskauskas D, Mohammed BM, et al. Abstract 402: Vitamin C Deficiency Impairs Cardiac Function and Post-infarction Survival in the Mouse. Circ Res. 2017;121(suppl_1):A402. doi:10.1161/res.121.suppl_1.402

42. Ginter E. Chronic vitamin C deficiency increases the risk of cardiovascular diseases. Bratisl Lek Listy. 2007;108(9):417421. Accessed December 3, 2021. http://bmj.fmed.uniba. sk/2007/10809-09.pdf

43. Piscaer I, Wouters EFM, Vermeer C, Janssens W, Franssen FME, Janssen R. Vitamin K deficiency: the linking pin between COPD and cardiovascular diseases? Respir Res. 2017;18(1):189. doi:10.1186/s12931-017-0673-z

44. Drapkina OM, Shepel RN. THE LINK BETWEEN VITAMIN B12 DEFICIENCY, RISK OF CARDIOVASCULAR DISEASES AND AGING PROCESS. Rational 
Pharmacotherapy in Cardiology. 2017;13(1):100-106. doi:10.20996/1819-6446-2017-13-1-100-106.

45. Hashmi O. Vitamin B deficiency may increase risk of lung cancer. Thorax. 2011;66(1):73. doi:10.1136/thx.2010.150235

46. Sousa MV, Amaral AG, Freitas JA, et al. Smoking accelerates renal cystic disease and worsens cardiac phenotype in Pkd1-deficient mice. Sci Rep. 2021;11(1):14443. doi:10.1038/s41598-021-93633-7

47.Liu Y, Li H, Wang J, et al. Association of Cigarette Smoking With Cerebrospinal Fluid Biomarkers of Neurodegeneration, Neuroinflammation, and Oxidation. JAMA Netw Open. 2020;3(10):e2018777. doi:10.1001/jamanetworkopen.2020.18777

48. Araujo I, Henriksen A, Gamsby J, Gulick D. Impact of Alcohol Abuse on Susceptibility to Rare Neurodegenerative Diseases. Front Mol Biosci. 2021;8:643273. doi:10.3389/fmolb.2021.643273

49. Loftfield E, Stepien M, Viallon V, et al. Novel Biomarkers of Habitual Alcohol Intake and Associations With Risk of Pancreatic and Liver Cancers and Liver Disease Mortality. J Natl Cancer Inst. 2021;113(11):1542-1550. doi:10.1093/jnci/djab078

50. Hamada K, Yoshimura K, Hirasawa Y, et al. Antibiotic Usage Reduced Overall Survival by over $70 \%$ in Non-small Cell Lung Cancer Patients on Anti-PD-1 Immunotherapy. Anticancer Res. 2021;41(10):4985-4993. doi:10.21873/anticanres.15312

51. Lu SSM, Mohammed Z, Häggström C, et al. Antibiotics Use and Subsequent Risk of Colorectal Cancer: A Swedish Nationwide Population-Based Study. J Natl Cancer Inst. 2021:djab125. doi:10.1093/jnci/djab125

52. Mak JWY, Sun Y, Wilson-O'Brien AL, et al. OP-0037 Childhood antibiotics are a risk factor for developing Crohn's disease. The ENIGMA international cohort study. J Gastroenterol Hepatol. 2021;36(S2):41-42. Accessed December 3, 2021. https://onlinelibrary.wiley.com/doi/epdf/10.1111/ jgh.15604

53. Han F, Zhao J, Zhao G. Prolonged Volatile Anesthetic Exposure Exacerbates Cognitive Impairment and Neuropathology in the 5xFAD Mouse Model of Alzheimer's Disease. J Alzheimers Dis. 2021;84(4):1551-1551. doi:10.3233/JAD-210374

54.Zhao Y, Liang G, Chen Q, et al. Anesthetic-induced neurodegeneration mediated via inositol 1,4,5-trisphosphate receptors. J Pharmacol Exp Ther. 2010;333(1):14-22. doi:10.1124/jpet.109.161562

55. Koponen M, Rajamaki B, Lavikainen P, et al. Antipsychotic Use and Risk of Stroke Among Community-Dwelling People With Alzheimer's Disease. J Am Med Dir Assoc. Published online October 28, 2021. doi:10.1016/j.jamda.2021.09.036

56. d'Errico A, Strippoli E, Vasta R, Ferrante G, Spila Alegiani $\mathrm{S}$, Ricceri F. Use of antipsychotics and long-term risk of parkinsonism. Neurol Sci. Published online October 15, 2021. doi:10.1007/s10072-021-05650-z

57.Pereira LC, Araújo VP, Campbell-Borges I. Persistent Parkinsonian Syndrome Induced By Radiotherapy
And Chemotherapy. Stereotact Funct Neurosurg. 2013 2013;91(suppl 1):172-172. Accessed December 3, 2021. https://www.karger.com/Article/Pdf/351990

58. Sahebnasagh A, Saghafi F, Azimi S, Salehifar E, Hosseinimehr SJ. Pharmacological Interventions for the Prevention and Treatment of Kidney Injury Induced by Radiotherapy: Molecular Mechanisms and Clinical Perspectives. Curr Mol Pharmacol. 2021;14. doi:10.2174/1874467214666210824123212

59. Xiao W, Huang J, Wang J, Chen Y, Hu N, Cao S. Occupational exposure to organic solvents and breast cancer risk: a systematic review and meta-analysis. Environ Sci Pollut Res Int. Published online October 22, 2021. doi:10.1007/s11356-021-17100-6

60.Sakizadeh M. Data for: Spatiotemporal variations and characterization of the chronic cancer risk associated with benzene exposure. Mendeley Data. Published online July 11, 2019. doi:10.17632/3nsph96w44.1

61. Meydan S, Altas M, Nacar A, et al. The protective effects of omega-3 fatty acid against toluene-induced neurotoxicity in prefrontal cortex of rats. Hum Exp Toxicol. 2012;31(11):1179-1185. doi:10.1177/0960327112457187

62. Amir S, Shah STA, Mamoulakis C, et al. Endocrine Disruptors Acting on Estrogen and Androgen Pathways Cause Reproductive Disorders through Multiple Mechanisms: A Review. Int J Environ Res Public Health. 2021;18(4):1464. doi:10.3390/ijerph18041464

63. Wen HJ, Chang TC, Ding WH, Tsai SF, Hsiung CA, Wang SL. Exposure to endocrine disruptor alkylphenols and the occurrence of endometrial cancer. Environ Pollut. 2020;267:115475. doi:10.1016/j.envpol.2020.115475

64. Tsatsakis A, Petrakis D, Nikolouzakis TK, et al. COVID-19, an opportunity to reevaluate the correlation between longterm effects of anthropogenic pollutants on viral epidemic/ pandemic events and prevalence. Food Chem Toxicol. 2020;141:111418. doi:10.1016/j.fct.2020.111418

65. Bogumil D, Wu AH, Stram D, et al. The association between ambient air pollutants and pancreatic cancer in the Multiethnic Cohort Study. Environ Res. 2021;202:111608. doi:10.1016/j.envres.2021.111608

66. Rodrigues SD, Ueda RM, Barreto AC, Zanini RR, Souza AM. How atmospheric pollutants impact the development of chronic obstructive pulmonary disease and lung cancer: A var-based model. Environ Pollut. 2021;275:116622. doi:10.1016/j.envpol.2021.116622

67. Sevim Ç, Çomaklı S, Taghizadehghalehjoughi A, et al. An imazamox-based herbicide causes apoptotic changes in rat liver and pancreas. Toxicol Rep. 2018;6:42-50. doi:10.1016/j.toxrep.2018.11.008

68. Georgiadis N, Tsarouhas K, Tsitsimpikou C, et al. Pesticides and cardiotoxicity. Where do we stand? Toxicol Appl Pharmacol. 2018;353:1-14. doi:10.1016/j.taap.2018.06.004

69. Dardiotis E, Aloizou AM, Sakalakis E, et al. Organochlorine pesticide levels in Greek patients with Parkinson's disease. Toxicol Rep. 2020;7:596-601. doi:10.1016/j.toxrep.2020.03.011 
70. Aloizou AM, Siokas V, Vogiatzi C, et al. Pesticides, cognitive functions and dementia: A review. Toxicol Lett. 2020;326:3151. doi:10.1016/j.toxlet.2020.03.005

71.Wallace DR, Spandidos DA, Tsatsakis A, Schweitzer A, Djordjevic V, Djordjevic AB. Potential interaction of cadmium chloride with pancreatic mitochondria: Implications for pancreatic cancer. Int J Mol Med. 2019;44(1):145-156. doi:10.3892/ijmm.2019.4204

72. Renieri EA, Safenkova IV, Alegakis AK, et al. Cadmium, lead and mercury in muscle tissue of gilthead seabream and seabass: Risk evaluation for consumers. Food Chem Toxicol. 2019;124:439-449. doi:10.1016/j.fct.2018.12.020

73. Akinyemi AJ, Miah MR, Ijomone OM, et al. Lead (Pb) exposure induces dopaminergic neurotoxicity in Caenorhabditis elegans: Involvement of the dopamine transporter. Toxicol Rep. 2019;6:833-840. doi:10.1016/j.toxrep.2019.08.001

74. Skalny AV, Timashev PS, Aschner M, et al. Serum Zinc, Copper, and Other Biometals Are Associated with COVID-19 Severity Markers. Metabolites. 2021;11(4):244. doi:10.3390/metabo11040244

75. Soares ATG, Silva AC, Tinkov AA, et al. The impact of manganese on neurotransmitter systems. J Trace Elem Med Biol. 2020;61:126554. doi:10.1016/j.jtemb.2020.126554

76. Ke T, Tsatsakis A, Santamaría A, et al. Chronic exposure to methylmercury induces puncta formation in cephalic dopaminergic neurons in Caenorhabditis elegans. Neurotoxicology. 2020;77:105113. doi:10.1016/j.neuro.2020.01.003

77. Wallace DR, Taalab YM, Heinze S, et al. Toxic-Metal-Induced Alteration in miRNA Expression Profile as a Proposed Mechanism for Disease Development. Cells. 2020;9(4):901. doi:10.3390/cells9040901

78. Karaulov AV, Renieri EA, Smolyagin AI, et al. Long-term effects of chromium on morphological and immunological parameters of Wistar rats. Food Chem Toxicol. 2019;133:110748. doi:10.1016/j.fct.2019.110748

79. Iftode A, Drăghici GA, Macașoi I, et al. Exposure to cadmium and copper triggers cytotoxic effects and epigenetic changes in human colorectal carcinoma HT-29 cells. Exp Ther Med. 2021;21(1):100. doi:10.3892/etm.2020.9532

80. Skalny AV, Lima TRR, Ke T, et al. Toxic metal exposure as a possible risk factor for COVID-19 and other respiratory infectious diseases. Food Chem Toxicol. 2020;146:111809.

\section{doi:10.1016/j.fct.2020.111809}

81. Tsatsakis A, Tyshko NV, Docea AO, et al. The effect of chronic vitamin deficiency and long term very low dose exposure to 6 pesticides mixture on neurological outcomes - A real-life risk simulation approach. Toxicol Lett. 2019;315:96-106. doi:10.1016/j.toxlet.2019.07.026

82. Tsiaoussis J, Antoniou MN, Koliarakis I, et al. Effects of single and combined toxic exposures on the gut microbiome: Current knowledge and future directions. Toxicol Lett. 2019;312:72-97. doi:10.1016/j.toxlet.2019.04.014

83. Karaulov AV, Smolyagin AI, Mikhailova IV, et al. Assessment of the combined effects of chromium and benzene on the rat neuroendocrine and immune systems. Environ Res. 2021:112096. doi:10.1016/j.envres.2021.112096

84. Kostoff RN, Briggs MB, Porter AL, et al. The underreported role of toxic substance exposures in the COVID-19 pandemic. Food Chem Toxicol. 2020;145:111687. doi:10.1016/j.fct.2020.111687

\section{CONFLICTS OF INTEREST}

The author has completed and submitted the ICMJE Form for Disclosure of Potential Conflicts of Interest and none was reported.

FUNDING

There was no source of funding for this research.

ETHICAL APPROVAL AND INFORMED CONSENT

Ethical approval and informed consent were not required for this study.

\section{DATA AVAILABILITY}

The data supporting this research are available from the Georgia Tech Library: http://hdl.handle.net/1853/62019 